\title{
Preparation and Characterization of Nanofibrillated Cellulose from Bamboo Fiber via Ultrasonication Assisted by Repulsive Effect
}

\author{
Zhijun Hu, Rui Zhai, Jing Li, Yan Zhang, and Jiang Lin \\ Zhejiang Province Collaborative Innovation Center of Agricultural Biological Resources Biochemical Manufacturing, \\ Zhejiang University of Science and Technology, Hangzhou 310023, China
}

Correspondence should be addressed to Zhijun Hu; huzhijun@zju.edu.cn

Received 7 September 2017; Accepted 19 November 2017; Published 26 December 2017

Academic Editor: Xiaofei Tian

Copyright (C) 2017 Zhijun Hu et al. This is an open access article distributed under the Creative Commons Attribution License, which permits unrestricted use, distribution, and reproduction in any medium, provided the original work is properly cited.

\begin{abstract}
Nanofibrillated celluloses (NFCs) have recently drawn much attention because of their exceptional physicochemical properties. However, the existing preparation procedures either produce low yields or severely degrade the cellulose and, moreover, are not energy efficient. The purpose of this study was to develop a novel process using ultrasonic homogenization to isolate fibrils from bamboo fiber (BF) with the assistance of negatively charged entities. The obtained samples were characterized by the degree of substitution (DS) of carboxymethyl, Fourier-transform infrared (FT-IR) spectroscopy, X-ray diffraction (XRD), thermogravimetric analysis, and transmission electron microscopy (TEM). The results showed that an NFC yield could be obtained above $70 \%$ through this route. The enzyme hydrolysis could enhance the surface charge of the fiber, and mechanical activation facilitates an increase in the DS. The disintegrating efficiency of the cellulose fibrils significantly depended on the input power of ultrasonication and the DS. FT-IR spectra confirmed the occurrence of the carboxymethylation reaction based on the appearance of the characteristic signal for the carboxyl group. From XRD analysis, it was observed that the presence of the carboxyl groups makes the isolation more efficient attributed to the ionic repulsion between the carboxylate groups of the cellulose chains.
\end{abstract}

\section{Introduction}

In recent years, much more attention has been paid to the sustainable, green, and environmental friendly materials because of the increasing public interest in environmental issues and the growing pressure from legislative institutions $[1,2]$. Cellulose is the most abundant renewable natural biopolymer on earth and is present in a wide variety of living species such as plants, animals, and some bacteria. Cellulose is a multifunctional raw material which can self-assemble into well-defined architectures at multiple scales, from nano- to microsize, and is expected to be able to replace many nonrenewable materials [3]. Cellulose does not occur as an isolated individual molecule in nature, but it is found as assemblies of individual cellulose chain-forming fiber cell wall. Higher plants form highly crystalline cellulose microfibrils, each of which consists of 30-40 fully extended and linear cellulose chains and are the elements with the second smallest width $(\sim 3 \mathrm{~nm})$ after single cellulose chains [4]. Plant cell walls are comprised of cellulose microfibrils filled with hemicelluloses and lignin. The microfibrils are so tightly hooked to one another by multiple hydrogen bonds, that their extraction has proven extremely difficult. By using harsh aqueous mechanical or chemical treatment, wood fiber can be degraded and opened into their substructural units giving a material such as microfiber and nanofiber.

The term "nanocellulose" generally refers to cellulosic materials having at least one dimension in the nanometer range. There are two major structures, namely, nanofibrillated cellulose (NFC) and nanocrystalline cellulose (NCC), as shown in Figure 1. The dissimilarity is related to their morphology. NCC can be regarded as the crystalline regions of the NFC. NCC, showing a short rod-like shape 100 to $200 \mathrm{~nm}$ in length and 4 to $25 \mathrm{~nm}$ in diameter, has been extensively 


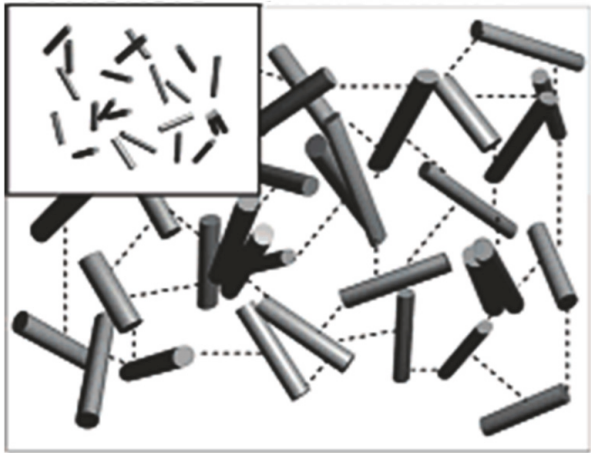

(a) Nanocrystalline cellulose (NCC) $L=100-300 \mathrm{~nm}$

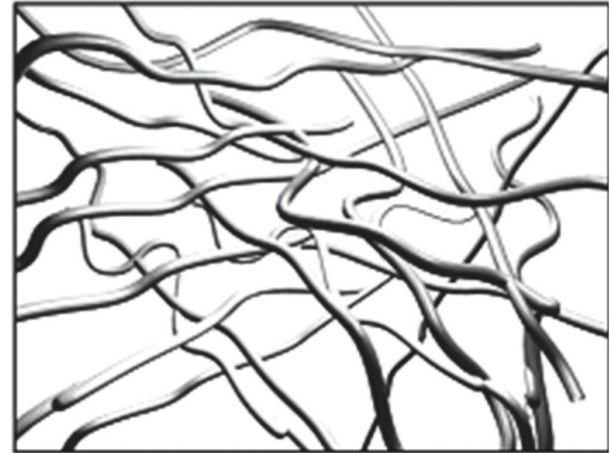

(b) Nanofibrillated cellulose (NFC) $L=$ several $\mu \mathrm{m}$

FIGURE 1: Diagram of NCC and NFC.

isolated by acid hydrolysis treatment [5]. NFC has a diameter below $100 \mathrm{~nm}$ and a length of several microns, which can be isolated by mechanical processes [6].

NFCs consist of a long web-like structure displaying exceptional mechanical properties [7] including a high Young's modulus, a high strength, and a very low coefficient of thermal expansion. NFCs have attracted great interests for its combination with a suitable matrix polymer for high-quality specialty applications of bio-based composites. Therefore, the development of effective methods for extracting NFCs from biomass has received an arising interest.

NFCs were normally produced by mechanical disintegration using either super-grinders [8], high-pressure homogenizers [9], high-pressure microfluidizer [10], or through cryocrushing [11]. However, the mechanical disintegration of the fibers into nanofibers often involves several passes through the disintegration device leading to the high energy consumption. Single disintegrating method suffers from two major drawbacks. The first challenge is the relatively low yields. The second is hydrophilic nature of cellulose causing irreversible agglomeration during drying and compounding in nonpolar matrices.

Combination of two or more methods presents a promising approach to overcome the above drawbacks. Researchers have combined various pretreatments with some suitable mechanical fibrillation technique, including the use of refining [12], enzymes [13], alkaline-acid treatment [14], ionic liquid treatment [15], and chemical modification [16, 17]. Among these methods, chemical modification, involving the addition of negatively charged entities at the microfibril surface, has attained considerable interest in the scientific community including the 2,2,6,6-tetramethylpiperidine-1oxyl (TEMPO) mediated oxidation [18] and carboxymethylation [19]. These are expected to result in NFC systems with smaller particle size distributions due to the high amount of charges as compared with other pretreatment processes $[20,21]$. The charges and their repulsive effect can greatly enhance the ease of separation and dispersion of individual microfibrils at a lower power consumption rate. However, a critical drawback associated with TEMPO is the negative environmental impact due to the use of sodium hypochlorite and sodium bromide as catalyst. Carboxymethylation has been used for the commercial production of sodium carboxymethyl cellulose (CMC), so its impact on the environment can be easily controlled or eliminated. Hence, carboxymethylation is a relatively environmentally friendly pretreatment for carrying out the fibrillation of cellulose fibers at low energies. Accordingly, this study investigates the extraction of nanofibrillated cellulose (NFC) from natural bamboo fiber under pulsed carboxymethylation.

Recently, the ultrasonic technique has been applied to isolate cellulose nanofibers and it has attracted considerable attention [22, 23]. The effect of ultrasonication in degrading polysaccharide linkages has been well described [24]. Ultrasonication is the application of sound energy to physical and chemical systems. Ultrasound produces its effects mainly through cavitation, which can introduce much effect such as intense shear forces, shock waves, and microjets, and generates localized hot spots with very high temperatures, pressures, and heating/cooling rates $[25,26]$. Such extreme environments provide a unique platform to break the strong cellulose interfibrillar hydrogen bonding, allowing nanofibers to be gradually disintegrated $[24,27]$.

Existing procedures for the production of NFC either result in low yields or severely degrade the cellulose and, moreover, are not environmentally friendly or energy efficient. In the present work, a novel route was developed for the preparation of water-redispersible NFC from bamboo fiber (BF). It consists of four steps including mechanical refining $(\mathrm{R})$, enzyme treatment (E), carboxymethyl modification (CM), and ultrasonic homogenization (S). The bamboo fiber was disintegrated mainly by the effect of ultrasonication with the assistance of negative charges. The resulting samples were analyzed by charge titration, Fourier-transform infrared (FTIR) spectroscopy, X-ray diffraction (XRD), thermogravimetric analysis (TGA), and transmission electron microscopy (TEM) to further investigate their chemical structure and morphology.

\section{Materials and Methods}

2.1. Materials. Raw bamboo was obtained from local farmers in Anji, Zhejiang province, China. Enzyme was obtained from Tianjin Changwei Biological Technology Co., Ltd., 




FiguRE 2: The individualization of bamboo fiber nanofibers from bamboo powders with typical laboratory-scale ultrasonication apparatus under neutral conditions.

and the cellulolytic activity was $1000 \mathrm{EGU} / \mathrm{g}$ (EGU means endoglucanase unit). Monochloroacetic acid (MCA) (sodium salt, purity $\geq 98 \%, M=116.48 \mathrm{~g} / \mathrm{mol}$ ) was purchased from Aladdin. All other chemicals used were of ACS reagent grade and purchased from Hangzhou Huipu Co., Ltd. Deionized water was used for all the experiments.

\subsection{Preparation of Bamboo Fibre (BF) and Nanocrystalline} Cellulose (NFC). The process of isolating nanocrystalline cellulose (NFC) from bamboo fiber consists of a series of chemical treatments and high-intensity ultrasonication according to the flowchart shown in Figure 2. First, treatment with acidified sodium chlorite was carried out at $75^{\circ} \mathrm{C}$ for $1 \mathrm{hr}$, which was repeated five times until the product became white. The major lignin was then removed following the method of Abe and Yano [28]. Next, an alkaline treatment with potassium hydroxide $(\mathrm{KOH})$ was conducted to remove the hemicelluloses, residual starch, and pectin. The obtained bleached bamboo fiber was labelled as BF.

The cell wall delamination of the BF was carried out in four steps: a refining (R) step to increase the accessibility of the cell wall to the subsequent endoglucanase treatment, an enzymatic treatment (E) step, a low degree of substitution carboxymethylation (CM) stage, and finally the treatment of the pulp slurry by ultrasonic homogenization $(\mathrm{S})$. The above samples were labeled as BF-R, BF-E, BF-CM, and BF$S$, respectively. In the enzymatic treatment, a total of $5 \mathrm{~g}$ $\mathrm{BF}, 200 \mathrm{~mL}$ acetate buffer solution $(\mathrm{pH}=4.8)$, and enzyme were added into a conical flask. Enzymatic hydrolysis was performed at $50^{\circ} \mathrm{C}$ and continuously stirred using a water bath. Subsequently, the samples were carboxymethylated, under specific conditions which are detailed in the literature [20]. In brief, the fibers were first dispersed in deionized water at 10,000 revolutions in an ordinary laboratory disperser. The fibers were then solvent-exchanged with ethanol by washing the fibers in ethanol four times with an intermediate filtration step. The fibers were then impregnated in a solution of $2 \%$ MCA in $500 \mathrm{~mL}$ isopropanol for $30 \mathrm{~min}$. This mixture was transferred to a $5 \mathrm{~L}$ reaction vessel equipped with reflux and containing a heated solution of $16.2 \mathrm{~g} \mathrm{NaOH}$ in a mixture of $3 / 5 \mathrm{v} / \mathrm{v}$ ethanol/isopropanol. The carboxymethylation reaction was allowed to continue for $1 \mathrm{hr}$. Finally, the samples were further treated by ultrasonic homogenization (S), after dispersion in $500 \mathrm{ml}$ of deionized water at a final concentration of $1.5 \% \mathrm{w} / \mathrm{w}$. The ultrasonic treatment was carried out in an ice bath to avoid overheating of the sample. The input power of ultrasonication was varied from 400 to $1200 \mathrm{~W}$. After ultrasonication, the suspension was concentrated by centrifugation at 5,000 rpm for $30 \mathrm{~min}$ [29]. The colloidal suspension of NFC was collected and freeze-dried for testing.

2.3. Determination of the Degree of Substitution (DS) of Carboxylate Group. Conductometric titration (CT) was used to determine the DS of carboxymethylated BF following the protocol [30]. 0.1 M aqueous hydrochloric acid $(\mathrm{HCl})$ solution was added to the fiber suspension and stirred with a magnetic bar. The conductivity of the suspension was measured upon titration with $0.1 \mathrm{M}$ aqueous $\mathrm{NaOH}$ solution, using a conductometer. The measurements were repeated three times for each sample. The titration curves showed the presence of a strong acid corresponding to the excess of $\mathrm{HCl}$ and a weak acid corresponding to the carboxylate content. The total amount of carboxymethyl (CM) groups and DS were calculated from

$$
\begin{aligned}
n_{\mathrm{CM}} & =\left(V_{1}-V_{0}\right) C_{\mathrm{NaOH}}, \\
\mathrm{DS} & =\frac{162 \times n_{\mathrm{CM}}}{m-58 n_{\mathrm{CM}}} \times 100 \%,
\end{aligned}
$$

where $n_{\mathrm{CM}}$ is the carboxymethyl content, $V_{1}$ and $V_{0}$ are the equivalent volumes of added $\mathrm{NaOH}$ solution, $C_{\mathrm{NaOH}}$ is the exact concentration of $\mathrm{NaOH}$ solution, and $m$ is the weight of dried product.

2.4. Determination of NFC Yield. The ultrasonic sample was collected and washed with sufficient deionized water by repeating the centrifugation and dilution processes until its $\mathrm{pH}$ was neutral, and then the sample was freeze-dried for $48 \mathrm{hr}$. The yield of NFCs was calculated as follows:

$$
Y=\frac{\left(m_{1}-m_{2}\right) V_{1}}{m V_{2}} \times 100 \%,
$$

where $Y$ is the yield of NFCs, $m_{1}$ is the total mass of vacuum freeze-dried NFC and weight bottle, $m_{2}$ is the mass of the weight bottle, and $m$ is the mass of $\mathrm{BF}$. 


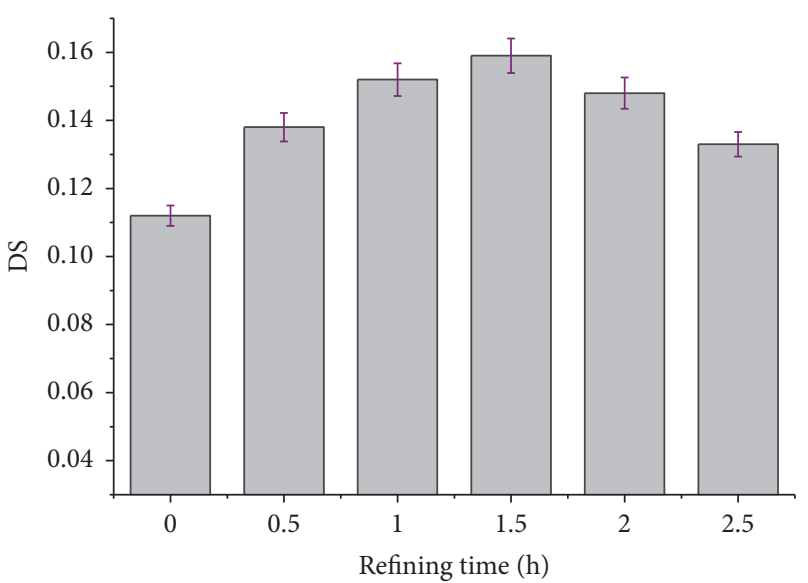

FIgURE 3: Effect of refining time on DS of cellulose.

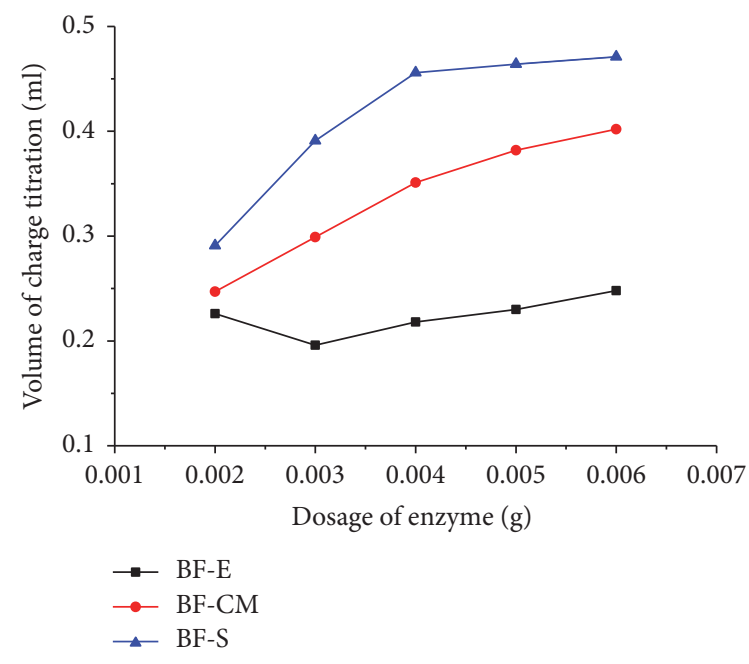

Figure 4: Effect of treatments on the surface charge.

As can be seen from Figure 3, with an increase of refining time, the DS of the cellulose increased from 0.112 (without refining) to 0.159 (after a refining time of $1.5 \mathrm{hr}$ ). This provides direct evidence that refining would be beneficial for the etherification reaction of cellulose. As the refining time extended beyond this point, the DS exhibited a slow decline. This may be due to effects such as the degradation of cellulose materials, the reversibility of the etherification reaction, and alkaline degradation of the cellulose. However, the DS of the refined cellulose was always greater than that of unrefined cellulose. The results demonstrated that mechanical treatment of the fiber can significantly improve the reactivity of cellulose. This is because mechanical activation can change the crystalline structure of cellulose, thus reducing its crystallinity, allowing etherified reagent to more easily penetrate into the interior cellulose, which is consistent with Huang et al.s study [31].

\subsection{Influence of Different Pretreatment Methods on the Surface} Charge. Three samples, named BF-E, BF-CM, and BF-S, were obtained following the route described in the Materials and Methods section (Section 2). The effect of the enzyme dosage on the surface charge is shown in Figure 4.

As can be seen in Figure 4, the surface charge of sample BF-E first decreased quickly and then increased slowly. The effect of the enzyme was first to hydrolyze the fibers and decrease the specific surface area of the fiber and hence reduce the number of exposed carboxyl groups on the fiber surface. With an increasing enzyme dosage, the enzyme hydrolyzed the fiber further, increasing the specific surface area of the fiber, and exposed more carboxyl groups, leading to an increase in the surface charge of the fibers. The surface charge of both BF-CM and BF-S first raised rapidly and then slowly with the increased dosage of enzyme. After carboxymethylation, the surface charge of the fibers increased significantly, indicating that the etherification enhanced the negative charge by increasing the number of carboxyl groups. Moreover, the final ultrasonic homogenization led to a remained unchanged. 


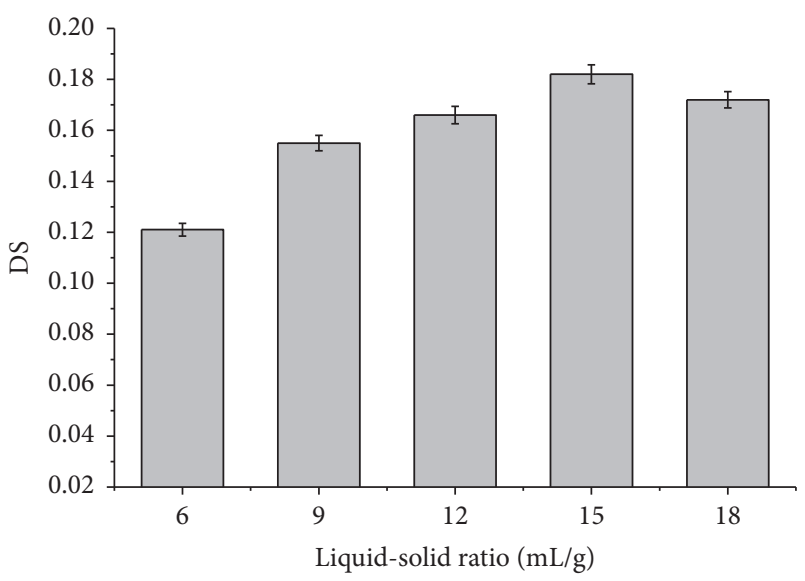

FIGURE 5: Effect of liquid-solid ratio on DS of cellulose.



Figure 6: Effect of ultrasonic time on NFCs yield.

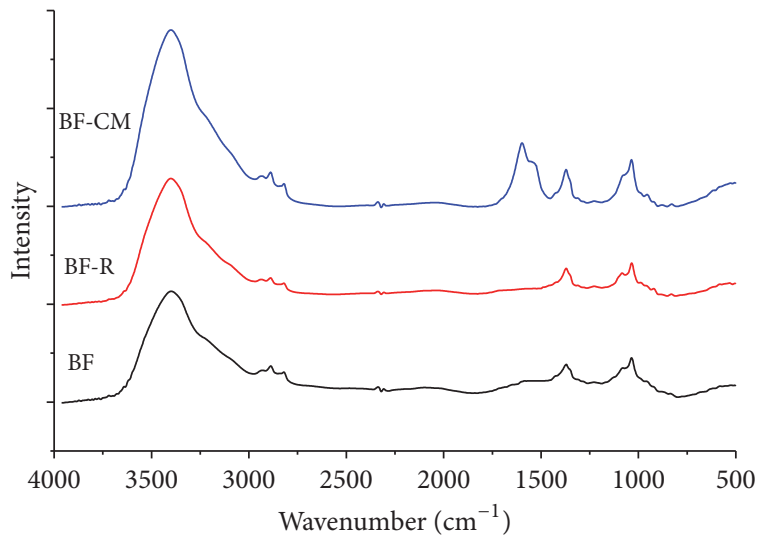

FIGURE 7: FT-IR of BF with different pretreatments. with the raw cellulose material, promoting the reaction and leading to an increase in the DS. The same phenomenon was also observed in Ye and Chen's study [32]. However, once the liquid-solid ratio was greater than $15 \mathrm{~mL} / \mathrm{g}$, an increase of the liquid-solid ratio only reduced the concentration of SCA, which did not benefit the flow state of the reaction system. Therefore, in the presence of low concentrations of SCA, the etherification of cellulose tended to decline.

3.4. Effect of Ultrasonication on the Yeild of NFCs. Ultrasonic homogenization is a crucial step affecting the defibrillation of the cellulose fiber. Ultrasonic treatment was conducted for time periods ranging from $20 \mathrm{~min}$ to $80 \mathrm{~min}$, with a constant power of $P 1\left(50 \% P_{\max }\right)$ and $P 2\left(70 \% P_{\max }\right)$. The yield of the NFC was calculated according to (4), and the results are shown in Figure 6.

As shown in Figure 6, the ultrasonication time had a significant effect on the NFC yield; with increasing ultrasonication time, the yield of NFC significantly increased. An NFC yield above $65 \%$ could be obtained through the route in the optimum conditions. When the ultrasonic time increased from $20 \mathrm{~min}$ to $50 \mathrm{~min}$, a significant increase in the NFC yield was observed. The yield reached a maximum of $72.46 \%$ with an ultrasonication time of $80 \mathrm{~min}$ and an input power of $70 \% P_{\max }$. The input power played an important role in the process of ultrasonic homogenization. The yield for 30 min with $P 2$ was higher than that of $80 \mathrm{~min}$ with $P 1$. This finding could be attributed to the breach of the inner crystallization area that needs sufficient power to be affected by ultrasonication. A similar result was reported in the literature [33].

3.5. FT-IR Analysis. To confirm successful carboxymethylation, the treated and untreated BF were characterized using FT-IR spectroscopy, and the results are shown in Figure 7.

The spectrum of the untreated $\mathrm{BF}$ showed the characteristic absorption bands of cellulose. A large band between $3,000 \mathrm{~cm}^{-1}$ and $3,800 \mathrm{~cm}^{-1}$ was attributed to the $\mathrm{OH}$ stretching vibrations, and the peak at $2,920 \mathrm{~cm}^{-1}$ was assigned to the $\mathrm{CH}$ stretching vibrations. A series of peaks between 1,300 and $1,500 \mathrm{~cm}^{-1}$ were associated with $\mathrm{OCH}$ deformation vibrations, $\mathrm{CH}_{2}$ bending vibrations, and the $\mathrm{CCH}$ and $\mathrm{COH}$ bending vibrations. Finally, the band ranging from 900 to $1,100 \mathrm{~cm}^{-1}$ mainly contained signals from CC stretching vibrations and $\mathrm{COH}$ and $\mathrm{CCH}$ deformation vibrations. Refining disintegration of the BF (BF-R) did not lead to a change in the FT-IR spectrum. However, the absorption peak intensities displayed obvious changes, and the absorption 


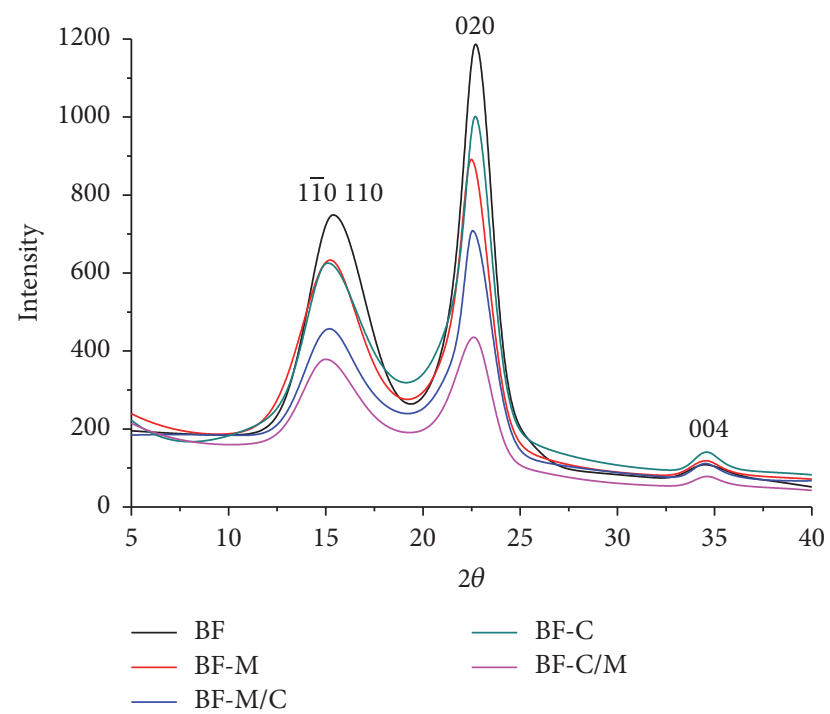

FIGURE 8: XRD diffractograms of BF samples.

peak attributed to the $\mathrm{OH}$ stretching vibrations became wider, indicating that, under the action of mechanical activation, the intermolecular hydrogen bonding of cellulose was abated, and the amount of free hydroxyls increased. Carboxymethylation of the BF-R (BF-CM) led to the appearance of a new signal at $1,598 \mathrm{~cm}^{-1}$, which was attributed to the asymmetric stretching vibration of the carboxylate group [34], confirming the successful carboxymethylation.

3.6. XRD Analysis. To investigate the effect of the sequence of chemical and mechanical treatments on the preparation of NFC, the crystallinities of the different samples were analyzed by X-ray diffraction. The samples were prepared through two routes. For route 1 , the BF was first treated by mechanical disintegration (BF-M) and then by chemical modification (BF- M/C). For route 2, the BF was first treated by chemical modification (BF-C) and then by mechanical disintegration $(\mathrm{BF}-\mathrm{C} / \mathrm{M})$. The results are shown in Figure 8.

The diffractogram of the untreated BF showed reflections of the cellulose lattice planes appearing at $2 \theta$ angles between $13^{\circ}$ and $17^{\circ}$ (110 and 110) and 22.3 (020) and 34.5 (004) [35]. The crystallinity $\chi_{\text {cr }}$ of the samples was estimated using (4) [36] with $I_{020}$ being the intensity of the 020 peak (amorphous and crystalline reflections at $22.3^{\circ}$ ) and $I_{\text {am }}$ being the intensity of the minimum between the 020 and 110 peaks.

$$
\chi_{\mathrm{cr}}=\frac{I_{020}-I_{\mathrm{am}}}{I_{020}} .
$$

According to (1), the highest value of crystallinity was obtained for raw BF (77.74\%), followed by BF-M (69.02\%), BF-M/C (66.99\%), BF-C (63.19\%), and BF-C/M (56.15\%). As can be observed, mechanical disintegration of the BF (see curve of BF-M) strongly decrease its crystallinity, and the crystallinity was not significantly affected by postchemical modification (BF- M/C), implying that mechanical disintegration had a stronger effect on crystallinity than chemical



Figure 9: TGA of (a) BF (black curve), (b) BF-E (red curve), (c) BF-CM (blue curve), and (d) BF-S (green curve).

modification. However, samples from route 2 (BF-C and BF$\mathrm{C} / \mathrm{M}$ ) exhibited a more pronounced reduction in crystallinity compared to those from route 1 . It can be concluded that the presence of the carboxylate groups made the mechanical isolation more efficient, as not only amorphous regions but also crystalline domains were affected by the treatment. The improved efficiency of the isolation of the fibrillated material could be attributed to the ionic repulsion between the carboxylate groups of the single cellulose chains [20].

3.7. Thermogravimetry Analysis (TGA). It is well-known that substituent groups have a significant influence on the thermal stabilities and derivatives of cellulose. Therefore, the native and treated samples were characterized by thermogravimetric analysis in order to obtain information about their thermal behaviors. Figure 9 presents TGA curves for the BF and differently treated samples. All the TGA curves showed a small initial weight loss between 50 and $150^{\circ} \mathrm{C}$, which corresponds to a mass loss of absorbed moisture. For the original $\mathrm{BF}$, a slight weight loss occurred at $300^{\circ} \mathrm{C}$ and a drastic loss at $300-390^{\circ} \mathrm{C}$ (see black curve), followed by slow weight losses up to $600^{\circ} \mathrm{C}$. These results are similar to those reported in previous publications [37]. For BF-E, a significant weight loss started at approximately $285^{\circ} \mathrm{C}$ and ended at $374^{\circ} \mathrm{C}$; the enzyme treatment slightly decreased the thermal stability. Carboxymethylation clearly reduced the thermal stability, showing a significant weight loss starting at approximately $245^{\circ} \mathrm{C}$. This finding is in agreement with results obtained from the thermal degradation of partially carboxymethylated cellulose [38]. For BF-S, most weight loss occurred in the range of $210-500^{\circ} \mathrm{C}$, and the degradation temperature was further decreased. Ultrasonication resulted in $\mathrm{BF}$ with a reduced molecular weight, an increased specific surface area, and more exposed active groups. Generally, the low molecular weight of cellulose fragments introduced into the outer surface of the cellulose crystals resulted in a decrease in thermal stability. These results were similar to those reported in previous publications [39]. 


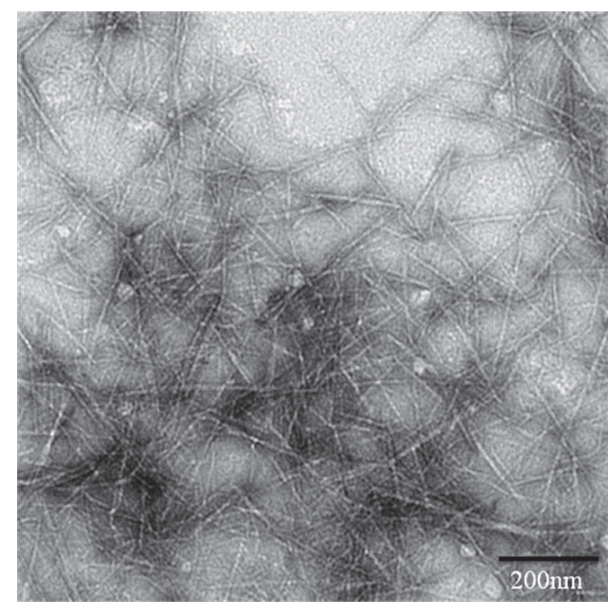

(a)

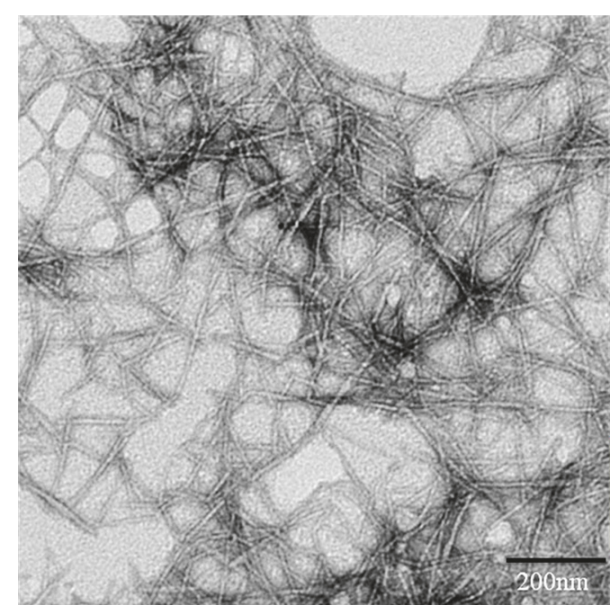

(b)

FIGURE 10: TEM images of water-redispersed samples: (a) DS $=0.135$ and (b) DS $=0.182$.

3.8. Morphological Structures of NFCs. The TEM images of the water-redispersed samples with different DS values were shown in Figure 10. As can be seen in Figure 10, most of individualized cellulose nanofibers were dispersed in water, and the cellulose nanofibers obtained were mainly $10-40 \mathrm{~nm}$ in width and a few microns in length. The sample with DS $=0.182($ Figure $10(\mathrm{~b}))$ presented better dispersion than that with DS $=0.135$ (Figure 10(a)). This revealed that the amount of negatively charged carboxyl groups played a critical role in the dispersion of NFC.

\section{Conclusions}

Nanofibrillated cellulose (NFC) from bamboo fiber was obtained by a combination of mechanical activation, enzyme treatment, carboxymethylation, and ultrasonic homogenization. It was encouraging that an NFC yield could be obtained above $70 \%$ through this route. Carboxymethylation was found to significantly increase the surface charge of the fibers. Ultrasonic homogenization led to a further augmentation of the surface charge by destroying the crystal structure of the fibers. Refining was able to activate the fiber by changing the crystalline structure of cellulose and allowing the etherified reagent to more easily penetrate into the cellulose interior, enhancing the efficiency of the etherification reaction of cellulose. The input power played a more significant role than the treating time in the process of ultrasonic homogenization attributed to the breach of the inner crystallization area requiring an adequate power of ultrasonication. FT-IR spectroscopy confirmed the occurrence of the carboxymethylation reaction in the modified $\mathrm{BF}$ by the appearance of characteristic signals at $1,598 \mathrm{~cm}^{-1}$. Although carboxymethylation modification did not significantly affect crystallinity, the presence of carboxylate groups was beneficial for destroying crystallinity of the posttreatment samples. The chemical modification or physical treatment decreased the degradation temperature of the fiber. The number of carboxyl groups, leading to the addition of negative charge, played a critical role in the dispersion of NFC.

\section{Conflicts of Interest}

The authors declare that they have no conflicts of interest.

\section{Authors' Contributions}

Zhijun Hu, Rui Zhai, Yan Zhang, Jing Li, and Jiang Lin designed the project. Zhijun Hu, Yan Zhang, and Rui Zhai performed the experiments and analyzed the data; Jing $\mathrm{Li}$ and Jiang Lin assisted in data processing and the manuscript preparation; and all authors contributed to the manuscript.

\section{Acknowledgments}

The authors are grateful for the support of the China Natural Science Foundation under Grant no. 51376162, the Key Industrial Project of Special Major Science and Technology of Zhejiang Province (no. 2013C01082), and the Zhejiang Province Collaborative Innovation Center of Agricultural Biological Resources Biochemical Manufacturing Foundation under Grant no. 2016KF0019.

\section{References}

[1] D. G. Gray, "Recent advances in chiral nematic structure and iridescent color of cellulose nanocrystal films," Nanomaterials, vol. 6, no. 11, pp. 213-221, 2016.

[2] H. P. S. A. Khalil, N. A. S. Aprilia, A. H. Bhat, M. Jawaid, M. T. Paridah, and D. Rudi, "A Jatropha biomass as renewable materials for biocomposites and its applications," Renewable \& Sustainable Energy Reviews, vol. 22, pp. 667-685, 2013.

[3] T. H. Wegner and P. E. Jones, "Advancing cellulose-based nanotechnology," Cellulose, vol. 13, pp. 115-118, 2014.

[4] A. Isogai, "Wood nanocelluloses: Fundamentals and applications as new bio-based nanomaterials," Journal of Wood Science, vol. 59, no. 6, pp. 449-459, 2013.

[5] B. Wang, M. Sain, and K. Oksman, "Study of structural morphology of hemp fiber from the micro to the nanoscale," Applied Composite Materials, vol. 14, no. 2, pp. 89-103, 2007. 
[6] L. Du, J. Wang, Y. Zhang, C. Qi, M. P. Wolcott, and Z. Yu, "Preparation and Characterization of Cellulose Nanocrystals from the Bio-ethanol Residuals," Journal of Synthetic Crystals, vol. 7, pp. 593-605, 2009.

[7] G. Siqueira, J. Bras, and A. Dufresne, "Cellulosic bionanocomposites: a review of preparation, properties and applications," Polymer, vol. 2, no. 4, pp. 728-765, 2010.

[8] A. F. Turbak, F. W. Snyder, and K. R. Sandberg, "Microfibrillated cellulose, a new cellulose product: properties, uses and commercial potential," Journal of Applied Polymer Science. Applied Polymer Symposium, vol. 37, pp. 815-827, 1983.

[9] T. Taniguchi, "Microfibrillation of natural fibrous materials," Journal of the Society of Materials Science Japan, vol. 45, pp. 472473, 1996.

[10] M. Pääkko, M. Ankerfors, H. Kosonen et al., "Enzymatic hydrolysis combined with mechanical shearing and highpressure homogenization for nanoscale cellulose fibrils and strong gels," Biomacromolecules, vol. 8, no. 6, pp. 1934-1941, 2007.

[11] A. Chakraborty, M. Sain, and M. Kortschot, "Cellulose microfibrils: a novel method of preparation using high shear refining and cryocrushing," Holzforschung, vol. 59, no. 1, pp. 135-107, 2005.

[12] W. Stelte and A. R. Sanadi, "Preparation and characterization of cellulose nanofibers from two commercial hardwood and softwood pulps," Industrial \& Engineering Chemistry Research, vol. 48, no. 24, pp. 11211-11219, 2009.

[13] M. Henriksson, G. Henriksson, L. A. Berglund, and T. Lindström, "An environmentally friendly method for enzymeassisted preparation of microfibrillated cellulose (MFC) nanofibers," European Polymer Journal, vol. 43, no. 8, pp. 3434-3441, 2007.

[14] A. Alemdar and M. Sain, "Isolation and characterization of nanofibers from agricultural residues-wheat straw and soy hulls," Bioresource Technology, vol. 99, no. 6, pp. 1664-1671, 2008.

[15] M. I. Voronova, T. N. Lebedeva, M. V. Radugin, O. V. Surov, A. N. Prusov, and A. G. Zakharov, "Interactions of water-DMSO mixtures with cellulose," Journal of Molecular Liquids, vol. 126, no. 1-3, pp. 124-129, 2006.

[16] D. da Silva Perez, S. Montanari, and M. R. Vignon, “TEMPOmediated oxidation of cellulose III," Biomacromolecules, vol. 4, no. 5, pp. 1417-1425, 2003.

[17] N. Siddiqui, R. H. Mills, D. J. Gardner, and D. Bousfield, "Production and characterization of cellulose nanofibers from wood pulp," Journal of Adhesion Science and Technology, vol. 25, no. 6-7, pp. 709-721, 2011.

[18] A. Benkaddour, K. Jradi, S. Robert, and C. Daneault, "Study of the effect of grafting method on surface polarity of tempooxidized nanocellulose using polycaprolactone as the modifying compound: esterificationversusclick-chemistry," Nanomaterials, vol. 3, no. 4, pp. 638-654, 2013.

[19] C. Eyholzer, A. Borges De Couraça, F. Duc et al., "Biocomposite hydrogels with carboxymethylated, nanofibrillated cellulose powder for replacement of the nucleus pulposus," Biomacromolecules, vol. 12, no. 5, pp. 1419-1427, 2011.

[20] L. Wågberg, G. Decher, M. Norgren, T. Lindström, M. Ankerfors, and K. Axnäs, "The build-up of polyelectrolyte multilayers of microfibrillated cellulose and cationic polyelectrolytes," Langmuir, vol. 24, no. 3, pp. 784-795, 2008.
[21] A. Tanaka, V. Seppänen, J. Houni, A. Sneck, and P. Pirkonen, "Nanocellulose characterization with mechanical fractionation," Nordic Pulp \& Paper Research Journal, vol. 27, no. 4, pp. 689-694, 2012.

[22] S. Xiao, R. Gao, Y. Lu, J. Li, and Q. Sun, "Fabrication and characterization of nanofibrillated cellulose and its aerogels from natural pine needles," Carbohydrate Polymers, vol. 119, pp. 202-209, 2015.

[23] Q. Zhang, M. Benoit, K. De Oliveira Vigier et al., "Pretreatment of microcrystalline cellulose by ultrasounds: Effect of particle size in the heterogeneously-catalyzed hydrolysis of cellulose to glucose," Green Chemistry, vol. 15, no. 4, pp. 963-969, 2013.

[24] P. C. S. F. Tischer, M. R. Sierakowski, H. Westfahl Jr., and C. A. Tischer, "Nanostructural reorganization of bacterial cellulose by ultrasonic treatment," Biomacromolecules, vol. 11, no. 5, pp. 1217-1224, 2010.

[25] M. J. Bussemaker, F. Xu, and D. Zhang, "Manipulation of ultrasonic effects on lignocellulose by varying the frequency, particle size, loading and stirring," Bioresource Technology, vol. 148, pp. 15-23, 2013.

[26] K. S. Suslick, Sonochemistry, Kirk-Othmer Encyclopedia of Chemical Technology, John wiley \& sons, New York, USA, 1998.

[27] B. W. Zeiger and K. S. Suslick, "Sonofragmentation of molecular crystals," Journal of the American Chemical Society, vol. 133, no. 37, pp. 14530-14533, 2011.

[28] K. Abe and H. Yano, "Comparison of the characteristics of cellulose microfibril aggregates of wood, rice straw and potato tuber," Cellulose, vol. 16, no. 6, pp. 1017-1023, 2009.

[29] C. Eyholzer, N. Bordeanu, F. Lopez-Suevos, D. Rentsch, T. Zimmermann, and K. Oksman, "Preparation and characterization of water-redispersible nanofibrillated cellulose in powder form," Cellulose, vol. 17, no. 1, pp. 19-30, 2010.

[30] R. W. Eyler, E. D. Klug, and F. Diephuis, "Determination of degree of substitution of sodium carboxymethylcellulose," Analytical Chemistry, vol. 19, no. 1, pp. 24-27, 1947.

[31] Z. Huang, X. Liang, L. Gao, H. Hu, and Z. Tong, "Graft copolymerization of mechanically activated sugarcane bagasse and acrylic acid (sodium acrylate)," Journal of the Chemical Industry and Engineering Society of China, vol. 60, pp. 15731580, 2009.

[32] Y. Ye and H. Chen, "Preparation of carboxymethylcellulose from steam exploded crop straw," Journal of the Chemical Industry and Engineering Society of China, vol. 60, pp. 1843$1849,2009$.

[33] S. Wang and Q. Cheng, "A novel process to isolate fibrils from cellulose fibers by high-intensity ultrasonication, part 1: process optimization," Journal of Applied Polymer Science, vol. 113, no. 2, pp. 1270-1275, 2010.

[34] L. T. Cuba-Chiem, L. Huynh, J. Ralston, and D. A. Beattie, "In situ particle film ATR FTIR spectroscopy of carboxymethyl cellulose adsorption on talc: Binding mechanism, $\mathrm{pH}$ effects, and adsorption kinetics," Langmuir, vol. 24, no. 15, pp. 80368044, 2008.

[35] J.-F. Sassi and H. Chanzy, "Ultrastructural aspects of the acetylation of cellulose," Cellulose, vol. 2, no. 2, pp. 111-127, 1995.

[36] L. Segal, J. Creely, A. Martin, and C. Conrad, "An empirical method for estimating the degree of crystallinity of native cellulose using the x-ray diffractometer," Textile Research Journal, vol. 29, no. 10, pp. 786-794, 1959.

[37] O. Ishida, D. Kim, S. Kuga, Y. Nishiyama, and R. M. Brown, "Microfibrillar carbon from native cellulose," Cellulose, vol. 11, no. $3 / 4$, pp. $475-480,2004$. 
[38] M. L. Leza, M. Cortazar, I. Casinos, and G. M. Guzmán, "Thermal degradation of partially carboxymethylated cellulose grafted with 4-vinylpyridine," Macromolecular Materials and Engineering, vol. 168, no. 1, pp. 195-203, 1989.

[39] S. Cui, S. Zhang, S. Ge, L. Xiong, and Q. Sun, "Green preparation and characterization of size-controlled nanocrystalline cellulose via ultrasonic-assisted enzymatic hydrolysis," Industrial Crops and Products, vol. 83, pp. 346-352, 2016. 

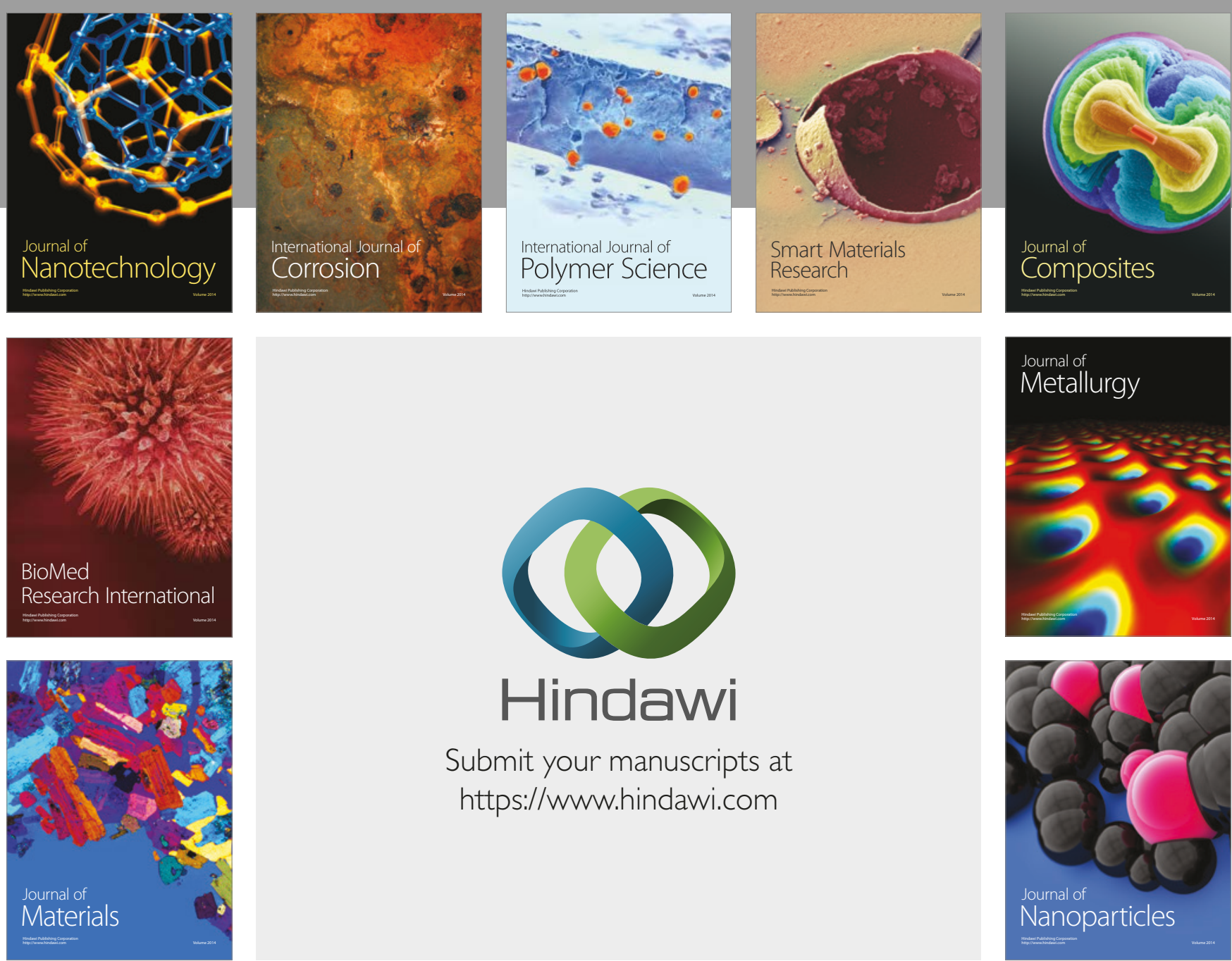

\section{Hindawi}

Submit your manuscripts at

https://www.hindawi.com


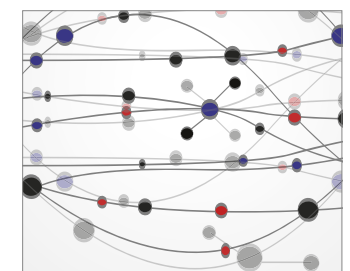

The Scientific World Journal
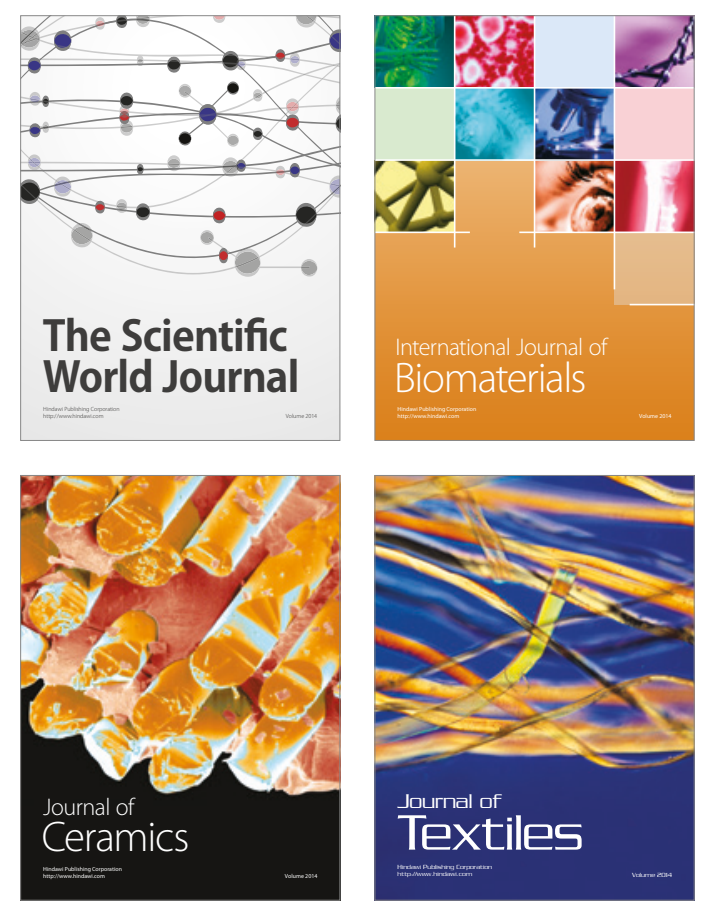